\title{
A Coupled Kinetic-Dynamic Model Characterising Cell Cycle Response to an Anti-Cancer Agent
}

\author{
M. I. Atari ${ }^{1}$, M. J. Chappell ${ }^{1}$, R. J. ERrington ${ }^{2}$, P. J. Smith ${ }^{2}$, N. D. Evans ${ }^{1}$ \\ ${ }^{1}$ School of Engineering, University of Warwick, Coventry, United Kingdom \\ ${ }^{2}$ University of Wales College of Medicine, Cardiff University, Cardiff, United Kingdom \\ E-mail: m.i.atari@warwick.ac.uk (M. I. Atari)
}

Sci Pharm. 2010; 78: 718

doi:10.3797/scipharm.cespt.8.POT03

\begin{abstract}
Topotecan (TPT) is a semi-synthetic derivative of the natural extract camptothecin, which has been found to act as an inhibitor of the DNA enzyme topoisomerase $\mathrm{I}$ in a specific and reversible fashion. The drug undergoes reversible hydrolysis from the pharmacologically active parent lactone form $\left(T_{P} T_{L}\right)$ to an inactive hydroxy acid form. In the cytoplasm the irreversible inactivation of TPT $T_{L}$ is catalysed by the enzyme aldehyde dehydrogenase (ALDH). Over-expression of the human breast cancer resistance protein (BCRP/ABCG2) has been linked to high levels of resistance to the anti-cancer agent TPT by promoting an active efflux pump mechanism. The expressions of both ALDH and BCRP have been experimentally identified in a large number of solid tumours and thus play an important role in clinical drug resistance of cancers. To investigate the catalytic reaction and efflux pump mechanism, a compartmental model for the in vitro uptake kinetics of TPT has been extended to better describe the drug activity and delivery of TPT $T_{L}$ to the DNA target as well as the catalysis by ALDH and the elimination of drug from the cytoplasm via the efflux pump. All unknown model parameters were uniquely estimated to a high level of confidence. Model simulations have been compared with live human breast cancer cells (MCF-7 cell line) data and found to give good qualitative agreement. The kinetic model has then been linked to a cell cycle model, based on [1], which facilitates analysis of the response of the growth of single cells in the presence and absence of TPT. Parameter estimation is performed using green fluorescent protein tagged cyclin B1 (cell cycle regulatory protein) data for the osteosarcoma cell line U-2 OS. Linking both models allows the study of drug perturbation to the cell cycle as well as in silico estimation and prediction of the relationship between the target binding and the dose, also permitting the effects of different expressions of the drug resistance protein and the ALDH enzyme. Such a coupled kinetic/dynamic model, once fully validated, has the potential for enhancing the design of optimal dosing regimens.

MIA is funded by the University of Warwick Vice Chancellor's PhD Scholarship.
\end{abstract}

[1] Pomerening JR, Sontag ED, Ferrell JE. Building a cell cycle oscillator: hysteresis and bistability in the activation of Cdc2. Nat Cell Biol. 2003; 5: 346-351. doi:10.1038/ncb954 